\title{
Constraints on dark matter and the shape of the Milky Way dark halo from the $511 \mathrm{keV}$ line
}

Y. Ascasibar ${ }^{1 \star}$, P. Jean ${ }^{2,3}$, C. Bœhm ${ }^{4,5}$ and J. Knödlseder ${ }^{2,3}$

${ }^{1}$ Harvard-Smithsonian Center for Astrophysics, 60 Garden St., Cambridge, MA 02138, USA

${ }^{2}$ Centre d'Étude Spatiale des Rayonnements, 9 avenue Colonel-Roche, BP 4346, 31028 Toulouse Cedex 4, France

3 Université Paul Sabatier, Toulouse 3, 118, Route de Narbonne, F-31062 Toulouse, France

${ }^{4}$ On leave LAPTH, UMR 5108, 9 chemin de Bellevue, BP 110, 74941 Annecy-Le-Vieux, France

${ }^{5}$ Physics department, Theory Division, CERN, CH-1211 Geneve 23, Switzerland

8 September 2006

N

\begin{abstract}
About one year ago, it was speculated that decaying or annihilating Light Dark Matter (LDM) particles could explain the flux and extension of the $511 \mathrm{keV}$ line emission in the galactic centre. Here we present a thorough comparison between theoretical expectations of the galactic positron distribution within the LDM scenario and observational data from INTEGRAL/SPI. Unlike previous analyses, there is now enough statistical evidence to put tight constraints on the shape of the dark matter halo of our galaxy, if the galactic positrons originate from dark matter. For annihilating candidates, the best fit to the observed $511 \mathrm{keV}$ emission is provided by a radial density profile with inner logarithmic slope $\gamma=1.03 \pm 0.04$. In contrast, decaying dark matter requires a much steeper density profile, $\gamma>1.5$, rather disfavoured by both observations and numerical simulations. Within the annihilating LDM scenario, a velocity-independent cross-section would be consistent with the observational data while a cross-section purely proportional to $v^{2}$ can be rejected at a high confidence level. Assuming the most simplistic model where the galactic positrons are produced as primaries, we show that the LDM candidate should be a scalar rather than a spin-1/2 particle and obtain a very stringent constraint on the value of the positron production cross-section to explain the $511 \mathrm{keV}$ emission. One consequence is that the value of the fine structure constant $\alpha$ should differ from that recommended in the CODATA. This is a very strong test for the LDM scenario and an additional motivation in favour of experiments measuring $\alpha$ directly. Our results finally indicate that an accurate measurement of the shape of the dark halo profile could have a tremendous impact on the determination of the origin of the $511 \mathrm{keV}$ line and vice versa.
\end{abstract}

Key words: Dark Matter - Galaxy: halo

\section{INTRODUCTION}

An emission line at $511 \mathrm{keV}$ was detected at the galactic centre three decades ago (Johnson et al. 1972). Its identification as an electron-positron annihilation line followed as soon as high-resolution spectrometers became available (Leventhal et al. 1978), but the origin of low-energy galactic positrons is still a matter of heated debate. The latest observations of the annihilation emission have been performed by the SPI spectrometer aboard the INTEGRAL ${ }^{1}$ satellite. A total flux of $\approx 10^{-3}$ photons $\mathrm{s}^{-1} \mathrm{~cm}^{-2}$ was measured,

\footnotetext{
* E-mail: yago@head.cfa.harvard.edu

1 INTEGRAL (International Gamma Ray Laboratory) is an ESA's gamma ray observatory launched in October 2002.
}

in agreement with previous estimates. The morphology of the galactic bulge emission could be fit by a Gaussian with $\sim 10{ }^{\circ} \mathrm{FWHM}$. A disc component was recently detected by INTEGRAL/SPI (Knödlseder et al. 2005) but this emission can be attributed to the $\beta^{+}$decay of the radioactive species ${ }^{26} \mathrm{Al}$ and ${ }^{44} \mathrm{Ti}$, which are produced by massive stars in the disc.

Several astrophysical sources have been proposed in the literature to explain the low-energy positrons from the bulge, such as radioactive nuclei expelled by stars (supernovae, hypernovae, novae, Wolf-Rayet stars and red giants) and collapsed objects (neutron stars or black holes). Nevertheless, most of these sources (see Knödlseder et al. 2005, and references therein) cannot account for the observed morphology, 
due to the large bulge-to-disc ratio of the emission, which suggests an old stellar population origin, unless rather elaborate mechanisms (e.g. jets, propagation) are invoked.

On the other hand, the presence of low-energy positrons could be explained by Dark Matter (DM) annihilations (Boehm et al. 2004) or decays (Hooper \& Wang 2004; Picciotto \& Pospelov 2005). The present paper focuses on such scenarios, which require light dark matter particles (i.e. with a mass $m_{\mathrm{dm}} \lesssim 100 \mathrm{MeV}$, depending on their exact nature) in order to reproduce the observational data.

The smallness of the DM mass might appear surprising to many. Indeed, not so long ago, most of the community thought that annihilating DM particles should be heavier than a few $\mathrm{GeV}$ because of the Lee-Weinberg limit (Lee \& Weinberg 1977), which states that if DM is a stable fermion coupled to heavy particles (such as the $\mathrm{Z}$ and $\mathrm{W}$ gauge bosons) then its mass should exceed that of the proton; otherwise it would overclose the universe. There are possible ways to evade the Lee-Weinberg limit though but the fact that theoretically motivated DM candidates, such as the lightest neutralino, were naturally very heavy did not encourage the community to investigate the lighter range.

The window for Light Dark Matter (LDM) particles suddenly opened when it was realized that scalar candidates with a mass from a few $\mathrm{MeV}$ to a few $\mathrm{GeV}$, coupled to heavy fermions $(F)$ or to light neutral particles (neutral gauge bosons $Z^{\prime}$, somewhat analogous to the $Z$ gauge boson), could also yield the observed relic density. However, the introduction of LDM particles immediately faces an embarassing problem: their annihilations are expected to produce too many low-energy gamma rays in our galaxy, compared to what has been observed. To be on the safe side, the presentday annihilation cross-section must be about five orders of magnitude (times $m_{\mathrm{MeV}}^{2}$, where $m_{\mathrm{MeV}} \equiv m_{\mathrm{dm}} c^{2} / 1 \mathrm{MeV}$ ) smaller than it was in the primordial universe (Boehm et al. 2004).

Such a condition can be easily satisfied if the channel associated to the exchange of heavy fermions is suppressed with respect to the one due to the new gauge boson. Indeed, the annihilation cross-section associated with a $Z^{\prime}$ exchange is proportional to the square of the DM velocity, which - in the Milky Way - is at least two or three orders of magnitude smaller than in the primordial universe (i.e. before DM became non-relativistic). Hence a velocity-dependent crosssection can satisfy both the relic density criterion and the gamma ray constraint. In contrast, the cross-section arising from heavy-fermion exchange does not depend at all on velocity; it remains constant at any epoch. The LDM scenario is thus viable if - as initially proposed - the $F$ exchange is at least five orders of magnitude (times $m_{\mathrm{MeV}}^{2}$ ) smaller than the $Z^{\prime}$ cross-section at early times. Note, though, that the contribution of the fermion exchange to the total annihilation cross-section could become dominant as velocities become non-relativistic.

Although the parameter space allowed for LDM is still quite broad, it is encouraging to find out that this model, originally built to be invisible (more precisely, to escape the low-energy gamma ray constraint) is able to explain the observed properties of the $511 \mathrm{keV}$ line without adding any new components nor changing the mass range initially proposed. Moreover, the existence of LDM particles might also explain several particle physics measurements (see e.g.


Figure 1. Dark matter annihilation through the exchange of a charged heavy fermion $F$ (left) or a neutral light gauge boson $Z^{\prime}$ (right).

Boehm \& Ascasibar 2004). The detailed analysis of the 511 $\mathrm{keV}$ line presented here provides new - and relatively tight - constraints on some of the LDM parameters, greatly enhancing the predictive capability of the model in other fields.

Conversely, independent confirmation of the LDM scenario from particle physics experiments would have important astrophysical consequences. In fact, if dark matter annihilation (light or otherwise) turns out to be the main source of galactic low-energy positrons, the observed morphology of the $511 \mathrm{keV}$ emission line would constitute an excellent probe of the shape of the Milky Way dark matter halo, whatever the exact nature of dark matter particles might be. The present study shows that several robust conclusions can already be derived from current INTEGRAL/SPI data.

Previous analyses (e.g. Jean et al. 2003) have shown that a point source can be ruled out a high confidence level. If positrons cannot travel a long distance before annihilating, that would seem to suggest that the galactic halo cannot be too 'cuspy'. On the other hand, a very flat profile would not match the observed morphology of the emission either, because its FWHM tells us that most of the positrons are generated within $1 \mathrm{kpc}$ from the galactic centre. According to Boehm \& Ascasibar (2004), the observed morphology is well described if the dark matter halo of the Milky Way follows a Navarro et al. (1997) profile. In this paper, we will attempt to constrain both the nature of dark matter and its distribution within the Milky Way halo as independently as possible.

Section 2 focuses on the main features of our dark matter model. The description of the Milky Way halo is discussed in Section 3 Section 4 is devoted to the comparison between the theoretical positron distribution and the observed flux. The results of our likelihood analysis are reported in Section 5] while Section [6] discusses additional issues, indirectly related to the present work. Finally, our main conclusions are briefly summarized in Section 7

\section{DARK MATTER CHARACTERISTICS}

Our study is based on the assumption that most galactic positrons originate from the decays or annihilations of LDM particles. The number density of positrons produced per unit of time is then dictated by the number density of dark matter particles, $n_{\mathrm{dm}}$, times their decay/annihilation rate into a pair $e^{+} e^{-}$. The latter are given by $\Gamma_{\mathrm{d}}$ or $\Gamma_{\mathrm{a}}=\left\langle\sigma v_{r}\right\rangle n_{\mathrm{dm}}$ * respectively, where $\left\langle\sigma v_{r}\right\rangle$ is the thermal average of the annihilation cross-section times the DM relative velocity. The two annihilation channels mentioned in the introduction are represented in Figure 1

Since the energy of the particles in the final state is imposed by kinematics, the only quantities that may appear in 
the formula of the annihilation cross-section are the masses of all particles involved, their couplings and the energy $E_{\mathrm{dm}}$ of the initial state. Since DM particles are nowadays nonrelativistic, $E_{\mathrm{dm}} \approx m_{\mathrm{dm}} c^{2}+m_{\mathrm{dm}} v^{2} / 2$, so the average crosssection can be re-written as $\left\langle\sigma v_{r}\right\rangle \approx a+b v^{2}+O\left(v^{4}\right)$, where both $v$ and $v_{r}$ are expressed in units of the speed of light, $c$.

In our case, the $F$-exchange cross-section has both an $a$ and a $b$-term; both of the same order of magnitude. In contrast, the $Z^{\prime}$ exchange gives rise to a pure velocity-dependent cross-section (i.e. $a=0$ ). A combination of both ingredients ( $Z^{\prime}$ and heavy fermions) thus provides a total annihilation cross-section with $a \neq b \neq 0$. Moreover, the crosssection through the $Z^{\prime}$ exchange depends on $m_{\mathrm{dm}}$, while the cross-section associated with the exchange of heavy particles is proportional to $1 / m_{F}^{2}$ or $m_{\mathrm{dm}}^{2} / m_{F}^{4}$, depending on whether DM is a spin- 0 or spin- $1 / 2$ particle, respectively (Boehm \& Favet 2004).

There are two independent constraints on the values of $a$ and $b$ (or more precisely on $\left\langle\sigma v_{r}\right\rangle$ ). On one hand, the relic density criterion imposes that the annihilation crosssection at the time of the chemical decoupling (i.e. when $T \sim m_{\mathrm{dm}}$ and $\left.v_{\mathrm{dm}} \sim c\right)$ is about $\left\langle\sigma v_{r}\right\rangle \sim 10^{-26} \mathrm{~cm}^{3} \mathrm{~s}^{-1}$ so that $\Omega_{\mathrm{dm}} h^{2} \sim 0.1$ nowadays. On the other hand, the amount of low-energy gamma rays produced at the present day in the centre of the Milky Way should not exceed the values observed by COMPTEL and EGRET (Strong et al. 2000). An estimate of the gamma ray flux generated by LDM annihilations has been computed by Boehm et al. (2004) in terms of $\left\langle\sigma v_{r}\right\rangle$, yielding ${ }^{2}$ an upper limit $\left\langle\sigma v_{r}\right\rangle \lesssim$ $10^{-31} \mathrm{~m}_{\mathrm{MeV}}^{2} \mathrm{~cm}^{3} \mathrm{~s}^{-1}$. Combining both constraints, we obtain

$\left\langle\sigma v_{r}\right\rangle_{\text {prim }} \approx a+b / 9 \sim 10^{-26} \mathrm{~cm}^{3} \mathrm{~s}^{-1}$

in the primordial universe (with $v_{\mathrm{dm}} \approx c / 3$ ) and

$\left\langle\sigma v_{r}\right\rangle_{\mathrm{now}} \approx a+v_{0}^{2} b \lesssim 10^{-31} \mathrm{~m}_{\mathrm{MeV}}^{2} \mathrm{~cm}^{3} \mathrm{~s}^{-1}$

in our Galaxy, where $v_{0} \sim 10^{-3} c$ and assuming that every DM annihilation into electron-positron instantaneously produces two photons with the maximal energy. Given that the photon production is expected to be through the final state radiation (i.e. a radiative correction to the annihilation process), we are clearly overestimating the gamma ray flux, making the constraint more stringent than it should be (see the discussion in Section 6.2].

In any case, it seems clear that, for $m_{\mathrm{dm}} \lesssim 100 \mathrm{MeV}$, the annihilation cross-section at the present time must be suppressed with respect to its primordial value in order to satisfy both the relic density (1) and the gamma ray (2) conditions, which lead lead to $a<10^{-31} \mathrm{~m}_{\mathrm{MeV}}^{2} \mathrm{~cm}^{3} \mathrm{~s}^{-1}$ and $b \sim 10^{-25} \mathrm{~cm}^{3} \mathrm{~s}^{-1}$. A velocity-dependent cross-section is thus necessary for LDM candidates below $\sim 100 \mathrm{MeV}$.

The gamma-ray constraint depends nevertheless on the shape of the dark halo profile. In particular, equation (2) above was derived from a NFW profile. Taking a flatter halo lessens these constraints and one finds that a

2 The flux obtained by Boehm et al. (2004) was actually overestimated by at least a factor four, since it was assumed that the number densities of dark matter particles and anti-particles were given by $n_{\mathrm{dm}}=n_{\mathrm{dm}^{\star}}=\rho_{\mathrm{dm}} / m_{\mathrm{dm}}$ instead of $n_{\mathrm{dm}}=n_{\mathrm{dm}^{\star}}=$ $\rho_{\mathrm{dm}} /\left(2 m_{\mathrm{dm}}\right)$. velocity-independent cross-section can match both the relic density and the gamma ray criteria if $m_{\mathrm{dm}}>20 \mathrm{MeV}$ Ahn \& Komatsu 2005). Hence, if the dark halo radial densiy profile of the Milky Way turns out to be flat, it would be possible to set $b=0$ and get rid of the $Z^{\prime}$.

In the following, the constants $a$ and $b$ will be normalized to $10^{-26} \mathrm{~cm}^{3} \mathrm{~s}^{-1}$, yielding the notation $a_{26}$ and $b_{26}$, respectively. For decaying LDM particles, the decay rate $\Gamma_{\mathrm{d}}$ will also be normalized to $10^{-26} \mathrm{~s}^{-1}$ (Hooper \& Wang 2004; Picciotto \& Pospelov 2005) yielding the notation $\Gamma_{26}$.

\section{THE MILKY WAY DARK HALO}

Pioneering analytical studies based on the spherical collapse formalism (Gunn \& Gott 1972; Gunn 1977) predicted that dark matter haloes ought to be described by a single powerlaw density profile, $\rho(r) \propto r^{-\gamma}$, with $\gamma$ ranging from 2 to 2.25 (e.g. Fillmore \& Goldreich 1984; Bertschinger 1985). Such result appeared quite compelling at the time, as it could straightforwardly explain the flatness of the rotation curves observed in spiral galaxies.

Later work based on numerical N-body simulations showed that the density profile was shallower than isothermal $(\gamma<2)$ as $r \rightarrow 0$, and steeper $(\gamma \approx 3)$ as $r \rightarrow \infty$. Typical values measured for the asymptotic logarithmic slope at the centre range from $\gamma=1$ (Navarro et al. 1997, hereafter NFW) to $\gamma=1.5$ (Moore et al. 1999, hereafter M99). The very existence of an asymptotic behaviour has recently been questioned by several studies (e.g. Power et al. 2003; Havashi et al. 2004; Navarro et al. 2004), in which the density profiles found in high-resolution simulations are reported to become progressively shallower inwards.

Despite the significant uncertainty on the shape of the density profile near the centre ${ }^{3}$, there is general agreement in that the dark matter distribution within a spherically symmetric halo can be well fitted by a 'universal' function with a small number of free parameters, and that the same functional form is valid for a broad range of halo masses and underlying cosmologies. Most of the analytical formulae proposed in the literature can be cast in the form

$\rho(r)=\frac{\rho_{0}}{\left(r / r_{0}\right)^{\gamma}\left[1+\left(r / r_{0}\right)^{\alpha}\right]^{(\beta-\gamma) / \alpha}}$,

where $\rho_{0}$ and $r_{0}$ are a characteristic density and radius of the halo, $\gamma$ is the asymptotic logarithmic slope at the centre, $\beta$ is the slope as $r \rightarrow \infty$ and $\alpha$ controls the exact shape of the profile in the intermediate regions around $r_{0}$.

Many different sets of values have been suggested for these parameters. The most notable discrepancies concern the value of $\gamma$. In particular, observed rotation curves of dwarf spiral and low surface brightness galaxies tend to favour flat profiles $(\gamma \approx 0)$, which has been often signalled as a genuine crisis of the CDM scenario (e.g. Flores \& Primack 1994; Moore 1994). Recent analyses show that observational data may actually be consistent with steeper profiles once the effects of inclination, non-circular orbits and triaxiality of the dark matter haloes are accounted for (Havashi et al.

3 Note that, in this region, numerical experiments are severely hampered by two-body relaxation (see e.g. Diemand et al. 2004) and discreteness effects (Binney 2004b). 
Table 1. Radial density profiles of the Milky Way dark halo considered in the present work, according to the parametrization given by expression [3].

\begin{tabular}{cccccc}
\hline MW & $\alpha$ & $\beta$ & $\gamma$ & $r_{0}[\mathrm{kpc}]$ & $\rho_{0}\left[\mathrm{GeV} \mathrm{cm}^{-3}\right]$ \\
\hline ISO & 2 & 2 & 0 & 4.0 & 1.655 \\
BE & 1 & 3 & 0.3 & 10.2 & 1.459 \\
NFW & 1 & 3 & 1 & 16.7 & 0.347 \\
M99 & 1.5 & 3 & 1.5 & 29.5 & 0.0536 \\
\hline
\end{tabular}

2004, 2005), but the controversy is still unresolved (e.g. Gentile et al. 2004; de Blok 2005).

In the Milky Way, it has been argued (Binnev \& Evans 2001, hereafter BE) that the microlensing optical depth towards the galactic centre reported by the MACHO collaboration (Alcock et al. 2000) would be incompatible with a $\gamma \gtrsim 0.3$ dark matter halo. A recent revision of the MA$\mathrm{CHO}$ results yields a lower optical depth, in somewhat better agreement with the values found by other experiments (see e.g. Sumi et al. 2005, for a recent review). Although a low optical depth could be consistent with steeper profiles, it is still unclear whether it would be compatible with the asymptotic slopes characteristic of numerical haloes (see e.g. Binnev 2004a).

On the other hand, the existence of a black hole at the centre of our galaxy (Gondolo \& Silk 1999) and the adiabatic contraction of the dark matter component due to the presence of baryons (Blumenthal et al. 1986) are expected to increase the central dark matter density, leading to a very steep profile in the innermost regions.

Most theoretical predictions of the gamma ray emission due to dark matter, including those performed in a supersymmetric framework (i.e. for heavy DM candidates), are based on 'cuspy' density profiles, since these maximize the expected flux. Given the present uncertainties, though, we have followed a completely different approach, trying to constrain the shape of the Milky Way dark matter halo as independently as possible from the precise nature of dark matter particles.

We therefore have considered four different models of the density profile of our galaxy, each one featuring a different asymptotic slope at the centre: in order of decreasing 'cuspiness', M99, NFW, BE and a non-singular isothermal sphere (hereafter ISO). Their corresponding parameters are summarized in Table 1 and the density and cumulative mass profiles are depicted in Figure 2 In addition, we also consider a family of models in which $\alpha$ and $\beta$ are fixed to 1 and 3 , respectively, while $\gamma$ is varied in uniform steps $\Delta \gamma=0.05$.

In all cases, the normalization of the models, $\rho_{0}$, is set by imposing a local dark matter density $\rho\left(r_{\odot}\right)=0.3 \mathrm{GeV} \mathrm{cm}^{-3}$, with $r_{\odot}=8.5 \mathrm{kpc}$. The characteristic radius $r_{0}$ has been chosen so that the virial radius and mass are $R_{\mathrm{vir}} \approx 260 \mathrm{kpc}$ and $M_{\mathrm{vir}} \approx 10^{12} \mathrm{M}_{\odot}$. Note that the ISO model can only approximately satisfy this condition.

The characteristic velocity of dark matter particles is also a necessary ingredient in our model of the Milky Way, as the LDM annihilation cross-section associated to $Z^{\prime}$ exchange explicitly depends on this quantity. In many studies, the rough estimate $v_{\mathrm{dm}} \sim 10^{-3} c$ is assumed to be accurate enough, but this is definitely not true for our present analysis. The positron emission arising from the velocity-

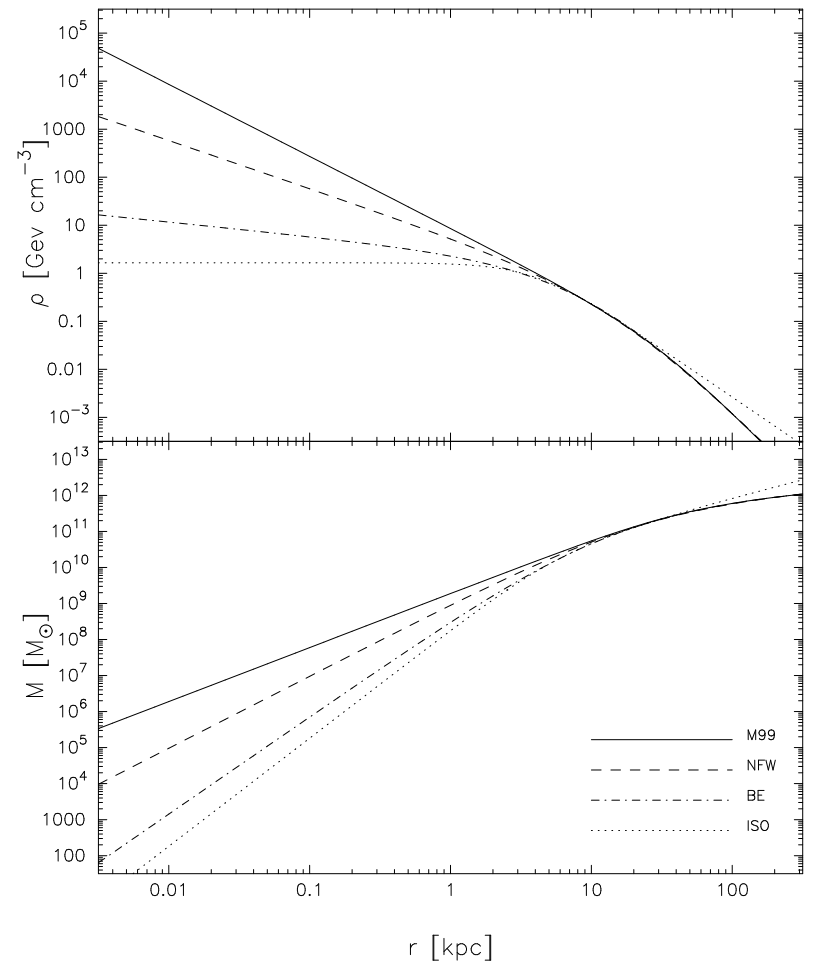

Figure 2. Top panel: Densty profiles considered in the present work. All of them are described by equation (3), with values of the parameters given in Table 1 Bottom panel: The corresponding cumulative mass profiles.

dependent term of the cross-section will be sensitive to the product $\rho^{2}(r) \sigma^{2}(r)$. Since we are trying to constrain the shape of the density profile, it is extremely important to properly account for the radial variation of the velocity dispersion.

Indeed, the velocity dispersion profile of dark matter haloes is known to change systematically with radius. As shown by Tavlor \& Navarro (2001), the phase-space density profile found in N-body simulations follows an approximate power law over several orders of magnitude in $r$. This result has been confirmed by Rasia et al. (2004) and Ascasibar et al. (2004) for haloes of very different mass. Following the latter, the density and velocity dispersion profiles would be subject to the phenomenological relationship

$\frac{\rho(r)}{\sigma^{3}(r)}=10^{1.46 \pm 0.04} \frac{\rho_{c}}{V_{\text {vir }}^{3}}\left(\frac{r}{R_{\text {vir }}}\right)^{-1.90 \pm 0.05}$

where $R_{\text {vir }}$ is the virial radius of the halo and we define $V_{\text {vir }}^{2} \equiv G M_{\text {vir }} / R_{\text {vir }}$. The cosmology-related quantities $\rho_{c}=1.36 \times 10^{11} \mathrm{M}_{\odot} \mathrm{Mpc}^{-3}$ and $M_{\mathrm{vir}} \approx \frac{4 \pi}{3} 100 \rho_{c} R_{\mathrm{vir}}^{3}$ are the critical density and virial mass corresponding to a "concordance' $\Lambda$ CDM universe $\left(\Omega_{\mathrm{m}}=0.3, \Omega_{\Lambda}=0.7, h=0.7\right)$. Substituting $R_{\mathrm{vir}}=260 \mathrm{kpc}$ for the Milky Way, we obtain $M_{\mathrm{vir}} \approx 10^{12} \mathrm{M}_{\odot}$ and $V_{\mathrm{vir}} \approx 130 \mathrm{~km} \mathrm{~s}^{-1}$. The velocity dispersion profile of our galaxy is thus expected to vary with radius as

$\frac{\sigma^{2}(r)}{c^{2}} \approx 6 \times 10^{-8}\left[\frac{\rho(r)}{1 \mathrm{GeV} \mathrm{cm}^{-3}}\left(\frac{r}{1 \mathrm{kpc}}\right)^{1.9}\right]^{2 / 3}$.

This formula has been used by Boehm \& Ascasibar 




Figure 3. Velocity dispersion profile obtained from the Jeans equation for each of the models in Table 1 Grey line on the top panel shows expression (5).

(2004) to estimate the characteristic velocity of dark matter particles. The radial dependence of the velocity dispersion significantly reduces the emission from the boson-exchange channel, being roughly equivalent to an effective flattening of the density profile.

However, expression (5) is a mere fit to the coarsegrained phase-space density profiles found in numerical simulations, and thus its validity has only been tested for 'cuspy' density profiles. A more self-consistent approach is followed in the present study, where we derive the velocity dispersion profiles from the spherically-symmetric Jeans equation,

$\frac{1}{\rho(r)} \frac{\mathrm{d}\left[\rho(r)\left\langle v_{\mathrm{ra}}^{2}(r)\right\rangle\right]}{\mathrm{d} r}+2 \beta(r) \frac{\left\langle v_{\mathrm{ra}}^{2}(r)\right\rangle}{r}=-\frac{G M(r)}{r^{2}}$,

where $v_{\text {ra }}$ is the radial component of the velocity and the anisotropy parameter $\beta(r)$ is defined as $\beta(r) \equiv 1-$ $\left\langle v_{\theta}^{2}(r)\right\rangle /\left\langle v_{\mathrm{ra}}^{2}(r)\right\rangle$. Assuming no radial infall, an isotropic velocity ellipsoid and vanishing velocity dispersion at infinity,

$\sigma^{2}(r)=\frac{3}{\rho(r)} \int_{r}^{\infty} \rho(r) \frac{G M(r)}{r^{2}}$.

The corresponding profiles are plotted in Figure 3 As can be readily seen, equation (5) provides a fair approximation for NFW and M99 models, but it is certainly not adequate for shallower density profiles.

\section{COMPARISON WITH SPI DATA}

According to the model outlined so far, the rate at which new positrons are created is

$\dot{n}_{\mathrm{e}^{+}}=n_{\mathrm{dm}} \Gamma$ where $\Gamma$ corresponds to the decay rate $\Gamma_{\mathrm{d}}$ for decaying dark matter and to the annihilation rate $\Gamma_{\mathrm{a}}=\left(a+b v_{\mathrm{dm}}^{2}\right) n_{\mathrm{dm}}$ * for annihilating LDM particles, with $n_{\mathrm{dm}^{\star}}$ being the number density of dark matter anti-particles.

These positrons will be relativistic at the moment of their creation $\left(E_{\mathrm{e}^{+}} \sim m_{\mathrm{dm}} c^{2}\right)$. However, they can efficiently lose their energy through collisional ionization or excitation in neutral Hydrogen and by interaction with plasma waves in ionized interstellar medium. We will make the approximation that they can only travel a short distance before becoming non-relativistic and annihilate with an electron at rest. Such an assumption is not very realistic for the outer regions of the galaxy, but it is perfectly reasonable for the galactic bulge, where most of the observed emission comes from.

Both OSSE (Kinzer et al. 2001), TGRS (Harris et al. 1998) and SPI (Churazov et al. 2005) measurements indicate that approximately 93 per cent of the positrons annihilate through positronium formation. In this channel, $3 / 4$ of the annihilations take place in the orthopositronium state, yielding 3 photons with $E<511 \mathrm{keV}$ each, while the remaining $1 / 4$ annihilate in the parapositronium state, producing 2 photons with $E=511 \mathrm{keV}$. The remaining 7 per cent that do not form a positronium annihilate directly into 2 photons with $E=511 \mathrm{keV}$. Consequently, the total number of 511 $\mathrm{keV}$ photons produced per unit time would be given by

$\dot{n}_{\gamma}=2(0.07+0.93 / 4) \dot{n}_{\mathrm{e}^{+}}=0.605 \dot{n}_{\mathrm{e}^{+}}$.

The predicted intensity distribution for any particular model of the Milky Way dark halo can be thus computed as the integral along the line of sight, as a function of galactic longitude $l$ and latitude $b$, of the emissivity $\dot{n}_{\gamma}(\boldsymbol{r})$,

$I(l, b)=\frac{1}{4 \pi} \int_{0}^{\infty} \dot{n}_{\gamma}(\boldsymbol{r}) \mathrm{d} s$,

where the spatial dependence arises through the radial density and relative velocity profiles $n_{\mathrm{dm}}(r)=n_{\mathrm{dm}^{\star}}(r)=$ $\rho_{\mathrm{dm}}(r) /\left(2 m_{\mathrm{dm}}\right)$ and $v_{\mathrm{dm}}^{2}(r) \approx \sigma^{2}(r)$. The total photon flux at the earth is simply

$\Phi=\int I(l, b) \mathrm{d} \Omega$.

Although the resulting sky map will obviously preserve the spherical symmetry of our models, the morphology of the emission (more specifically, its concentration) depends on the shape of the Milky Way halo as well as on the DM annihilation cross-section or decay rate ${ }^{4}$. Observations are mostly sensitive to the details of the central part, where the intensity of the $511 \mathrm{keV}$ line is highest. Dark matter in the outermost regions can make a significant contribution to the total flux, but the intensity of the emission is so low that it is difficult to discriminate from the instrumental background.

In order to compare with observational data, intensity maps $I(l, b)$ have been computed for $|l|<60^{\circ}$ and $|b|<50^{\circ}$. The total flux within this area is denoted by $\Phi_{\text {tot }}$. However, a fairer comparison with the flux measured by the satellite is given by the central $33^{\circ}(\sim 1$ steradian $)$. We shall quote this

4 Note that the precise distribution of baryons and their chemical composition should also be included in a realistic model of positron propagation. 
Table 2. Theoretical photon fluxes (in $\mathrm{cm}^{-2} \mathrm{~s}^{-1}$ ) expected for different halo profiles and DM type.

\begin{tabular}{cccc}
\hline DM & MW & $\Phi_{\text {tot }} m_{\mathrm{MeV}}^{2}$ & $\Phi_{\text {cen }} m_{\mathrm{MeV}}^{2}$ \\
\hline$\Gamma_{d}$ & ISO & $0.0459 \Gamma_{26}$ & $0.0201 \Gamma_{26}$ \\
& BE & $0.0439 \Gamma_{26}$ & $0.0195 \Gamma_{26}$ \\
& NFW & $0.0478 \Gamma_{26}$ & $0.0232 \Gamma_{26}$ \\
& M99 & $0.0512 \Gamma_{26}$ & $0.0262 \Gamma_{26}$ \\
$a$ & ISO & $5.13 a_{26}$ & $3.175 a_{26}$ \\
& BE & $5.21 a_{26}$ & $3.325 a_{26}$ \\
& NFW & $9.52 a_{26}$ & $7.487 a_{26}$ \\
& M99 & $26.6 a_{26}$ & $24.5 a_{26}$ \\
$b$ & ISO & $1.92 \times 10^{-6} b_{26}$ & $1.12 \times 10^{-6} b_{26}$ \\
& BE & $1.87 \times 10^{-6} b_{26}$ & $1.11 \times 10^{-6} b_{26}$ \\
& NFW & $2.93 \times 10^{-6} b_{26}$ & $2.10 \times 10^{-6} b_{26}$ \\
& M99 & $5.90 \times 10^{-6} b_{26}$ & $4.97 \times 10^{-6} b_{26}$ \\
\hline
\end{tabular}

flux as $\Phi_{\text {cen }}$. The values of $\Phi_{\text {tot }}$ and $\Phi_{\text {cen }}$ expected for each combination of dark matter type and radial density profile are given in Table 2

Our analysis has been performed on the December 10, 2004 public INTEGRAL data release, which consists of $\approx 309$ days of observations. In order to reduce systematic uncertainties in the analysis, we exclude observation periods with strong instrumental background fluctuations ${ }^{5}$. The total effective exposure time after cleaning is $15.3 \mathrm{Ms}$. The exposure is quite uniform in the central regions of our Galaxy $\left(|l|<50^{\circ}\right.$ and $\left.|b|<30^{\circ}\right)$.

We use a maximum likelihood algorithm to compare the theoretical sky maps with the INTEGRAL/SPI data. This method has already been applied to SPI data to characterize the morphology of the annihilation. A detailed description can be found in Knödlseder et al. (2005).

Briefly, the normalization of each theoretical model is fitted to reproduce the measured rate in the 507.5 $514.5 \mathrm{keV}$ energy range, taking into account an instrumental background model, the pointings history and the spatial and energy response functions of SPI. Normalized maps have been convolved with the response function, providing the expected number of counts in each detector as a function of the pointing periods. We then find the intensity that maximizes the log likelihood. We subtract from this log likelihood $L_{1}$ the log likelihood $L_{0}$ that is calculated under the hypothesis that there is no $511 \mathrm{keV}$ source. Multiplication by a factor of 2 provides the maximum log-likelihood ratio, $\operatorname{MLR}=2\left(L_{1}-L_{0}\right)$.

Results of the model-fitting procedure are presented in Table 3 and Table 4 As in the theoretical models, $\Phi_{\text {tot }}$ is the total flux of the map, integrated over the whole solid angle, while $\Phi_{\text {cen }}$ is restricted to an aperture of $33^{\circ}$. When comparing two models, the one with the largest MLR can be said to explain the data better than the other, although differences $\Delta_{\mathrm{MLR}}<10$ are, in principle, not very significant.

Strictly speaking, the posterior probability distribution for the flux, given the data and a particular model, is given by:

5 These background variations are generally due to solar flares or exit and entry of the observatory in radiation belts.
Table 3. Results of the model-fitting analysis. Fluxes in units of $10^{-3} \mathrm{~cm}^{-2} \mathrm{~s}^{-1}$.

\begin{tabular}{ccccc}
\hline DM & MW & $\Phi_{\text {tot }}$ & $\Phi_{\text {cen }}$ & MLR \\
\hline$\Gamma_{d}$ & ISO & $6.82 \pm 0.58$ & $2.95 \pm 0.25$ & 135.2 \\
& BE & $7.23 \pm 0.57$ & $3.18 \pm 0.25$ & 167.3 \\
& NFW & $7.36 \pm 0.46$ & $3.53 \pm 0.22$ & 261.2 \\
& M99 & $6.86 \pm 0.37$ & $3.48 \pm 0.19$ & 332.0 \\
$a$ & ISO & $5.55 \pm 0.33$ & $3.40 \pm 0.20$ & 282.8 \\
& BE & $4.98 \pm 0.27$ & $3.16 \pm 0.17$ & 353.6 \\
& NFW & $2.49 \pm 0.11$ & $1.95 \pm 0.09$ & 459.9 \\
& M99 & $0.83 \pm 0.04$ & $0.76 \pm 0.04$ & 339.2 \\
$b$ & ISO & $6.00 \pm 0.38$ & $3.46 \pm 0.22$ & 258.3 \\
& BE & $5.76 \pm 0.32$ & $3.40 \pm 0.19$ & 305.7 \\
& NFW & $3.61 \pm 0.18$ & $2.57 \pm 0.13$ & 422.4 \\
& M99 & $1.57 \pm 0.07$ & $1.32 \pm 0.06$ & 430.0 \\
\hline
\end{tabular}

Table 4. MLR and total flux (normalized to $10^{-3} \mathrm{~cm}^{-2} \mathrm{~s}^{-1}$ ) for the combined models $\phi_{a} I_{a}+\phi_{b} I_{b}$, where $I_{a}$ and $I_{b}$ are the intensity distributions associated with $a$ and $b$-terms, respectively.

\begin{tabular}{crrcc}
\hline DM & \multicolumn{1}{c}{$\phi_{a}$} & \multicolumn{1}{c}{$\phi_{b}$} & $\Phi_{\text {cen }}$ & MLR \\
\hline ISO & $28.07 \pm 2.68$ & $-26.35 \pm 2.85$ & 1.72 & 368.1 \\
BE & $15.11 \pm 1.32$ & $-13.95 \pm 1.53$ & 1.16 & 437.1 \\
NFW & $2.42 \pm 0.39$ & $-0.67 \pm 0.53$ & 1.75 & 461.4 \\
M99 & $-1.00 \pm 0.16$ & $2.82 \pm 0.25$ & 1.82 & 464.8 \\
\hline
\end{tabular}

$P(\Phi \mid D, M) \mathrm{d} \Phi=\frac{P(D \mid M, \Phi) P(\Phi \mid M) \mathrm{d} \Phi}{P(D \mid M)}$,

where $D$ is the data, $M$ is the model and $\Phi$ is the value of the flux, which can be regarded as a vector in the two-parameter models. $P(D \mid M, \Phi)$ is the likelihood of the data given $M$ and $\Phi, P(\Phi \mid M) \mathrm{d} \Phi$ is the prior probability distribution of the flux, and $P(D \mid M)$ is the Bayesian evidence of model $M$,

$P(D \mid M)=\int P(D \mid M, \Phi) P(\Phi \mid M) \mathrm{d} \Phi$.

The Bayesian evidence provides a measure of how well the model explains the data. More precisely, model $M_{1}$ can be considered $P\left(D \mid M_{1}\right) / P\left(D \mid M_{0}\right)$ times more likely than model $M_{0}$. Since the likelihood $P(D \mid M, \Phi)$ is strongly peaked around the best-fitting value of $\Phi$, the evidence is not very sensitive to the precise shape assumed for the prior. Morevoer, for practical purposes we can approximate $P(\Phi \mid D, M)$ by a Gaussian centered around the best-fitting $\Phi$ with $\sigma \approx \Delta \Phi$, so that the evidence can be computed as

$P(D \mid M) \propto e^{\mathrm{MLR}} \sqrt{2 \pi} \Delta \Phi$

for the models with one parameter, and

$P(D \mid M) \propto e^{\mathrm{MLR}} 2 \pi \Delta \phi_{a} \Delta \phi_{b}$

for the composite models in Table 4

It is evident from the figures in Table 3 that we can neglect the effect of $\Delta \Phi$ when comparing two models. In other words, a fair approximation to the Bayes' factor can be obtained by simply comparing the likelihood ratios,

$\log \frac{P\left(D \mid M_{1}\right)}{P\left(D \mid M_{0}\right)} \approx(\mathrm{MLR})_{1}-(\mathrm{MLR})_{0}$.

The model that best fits the INTEGRAL/SPI is a NFW profile with constant cross-section. This model closely 
ressembles the halo model $H^{\prime}$ of Knödlseder et al. (2005), with similar values of the best-fitting flux and MLR. All other models in Table 3 have $\Delta_{\text {MLR }}>20$ with respect to the $a$-term NFW or any of the bulge and halo models in Knödlseder et al. (2005) that satisfactorily explain the observed morphology of the $511 \mathrm{keV}$ emission. In frequentist terms, all the other density profiles and dark matter types listed in Table 3 can be rejected at the $\left(1-e^{-20}\right)$ confidence level, or $\sim \sqrt{20} \sigma$.

A rigorous Bayesian comparison between the one- and two-parameter models would require an extensive discussion of the priors. However, we note that linear combinations of the $a$-term and $b$-term models (c.f. Table 4) lead to marginal improvements of the MLR, but they provide non-physical solutions where one of the fluxes is negative. When the fit is constrained to non-negative values, zero fluxes are obtained for the negative coefficients, and the results quoted in Table 3 are reproduced for the other component. We therefore conclude that two-parameter models fail to provide a better explanation of the observational data.

\section{RESULTS}

\subsection{The nature of dark matter}

Comparing the best-fitting flux in Table 3 for a NFW profile with an $a$-term with our theoretical prediction in Table 2 we obtain

$a_{26}=(2.6 \pm 0.12) \times 10^{-4} m_{\mathrm{MeV}}^{2}$.

This result is valid for any kind of candidates, as long as the annihilation cross-section is parameterized as we have done in the present work and the galactic positrons are produced as primaries.

Equation (17) becomes comparable with (1) when $m_{\mathrm{dm}} \simeq 100 \mathrm{MeV}$. This confirms our previous conclusions from the gamma ray constraints, i.e. that the LDM scenario requires both an $a$ and $b$-term when $m_{\mathrm{dm}}<100 \mathrm{MeV}$.

To explain the $511 \mathrm{keV}$ emission line, candidates heavier than $100 \mathrm{MeV}$ would require a (velocity-independent) annihilation cross-section into a pair electron-positron that is well above the relic density requirement. Heavy candidates must thus produce positrons as secondaries to be a possible solution. However, one would expect an overproduction of gamma rays in the Milky Way in such case, unless there existed a channel that lead to a large production of positrons and a low production of gamma rays. This is why we advocate for LDM particles with $m_{\mathrm{dm}}<100 \mathrm{MeV}$ in order to explain the origin of galactic positrons.

For a scalar particle, the F-exchange channel yields

$\sigma v_{r}=\frac{c}{4 \pi} \sqrt{1-\left(\frac{m_{\mathrm{f}}}{m_{\mathrm{dm}}}\right)^{2}}\left[1-\left(\frac{m_{\mathrm{f}}}{m_{\mathrm{dm}}}\right)^{2}+v_{\mathrm{dm}}^{2}\right] \frac{c_{l}^{2} c_{r}^{2}}{m_{F}^{2}}$

where $m_{\mathrm{f}} \ll m_{\mathrm{dm}}$ is the mass of the fermions in the final state, $m_{F}$ is the mass of the heavy fermion that is exchanged during the annihilation and the quantities $c_{l}$ and $c_{r}$ correspond to the couplings.

Neglecting $m_{f}$ compared to $m_{\mathrm{dm}}$, we find

$a \simeq c \frac{c_{l}^{2} c_{r}^{2}}{4 \pi m_{F}^{2}} \Rightarrow a_{26} \simeq 9.32 \times 10^{3} c_{l}^{2} c_{r}^{2}\left(\frac{m_{F}}{100 \mathrm{GeV}}\right)^{-2}$
Substituting (19) into (17) yields

$\frac{m_{F}}{100 \mathrm{GeV}} \simeq 6 \times 10^{3} \frac{c_{l} c_{r}}{m_{\mathrm{MeV}}}$,

where the two couplings $c_{l}$ and $c_{r}$ are expected to be lower than unity (a few units at most).

For $c_{l} \approx c_{r} \approx 1$, one obtains $m_{F} \approx 6-O(600) \mathrm{TeV}$ (for $1 \lesssim m_{\mathrm{MeV}} \lesssim 100$ ). This is obviously out of reach for past and forthcoming colliders. However, for smaller but more realistic values of the couplings, $m_{F}$ could be within the range of next colliders. According to equation (20), a mass $m_{F} \in[100 \mathrm{GeV}, O(\mathrm{TeV})]$ would correspond to $c_{l} c_{r} \in 1.67 \times\left[10^{-4}, 10^{-3}\right] m_{\mathrm{MeV}}$. Those are not particularly small couplings (especially for $m_{\mathrm{MeV}} \sim 100 \mathrm{MeV}$ ) so there might be a signature of the LDM scenario in the next generation of accelerators, and in particular at LHC if the $F$ particles associated with quarks $\left(F_{q}\right)$ are not too heavy and their couplings not too small. Note that smaller couplings $e-F_{e}-d m$ (leading to $m_{F e}<100 \mathrm{GeV}$ ) are forbidden due to the absence of signal in past colliders (notably LEP).

So far we have considered scalar LDM particles. However, fermionic candidates (either Dirac or Majorana) have also been suggested (Boehm \& Favet 2004). The difference with respect to the scalar case would be that, for a Dirac fermion, the cross-section would be given by

$a_{\mathrm{D}} \sim \frac{c}{32 \pi}\left(c_{l}^{2}+c_{r}^{2}\right)^{2} \frac{m_{\mathrm{dm}}^{2}}{m_{F}^{4}}$

instead of (19), while for Majorana particles,

$a_{\mathrm{M}} \sim \frac{c}{8 \pi}\left(c_{l}^{2} c_{r}^{2}\right) \frac{m_{\mathrm{dm}}^{2}}{m_{F}^{4}}$,

where $F$ now denotes a scalar.

Using equation (17), we obtain that the mass $m_{F}$ should be

$\frac{m_{F}}{100 \mathrm{GeV}} \sim 0.145 \sqrt{\left(c_{l}^{2}+c_{r}^{2}\right)}$

for Dirac dark matter particles and

$\frac{m_{F}}{100 \mathrm{GeV}} \sim 0.206 c_{l} c_{r}$

for Majorana candidates.

Assuming realistic values for the couplings (i.e. $c_{l, r}<$ $O(1)$ ), we find that the $F$ mass is much smaller than $100 \mathrm{GeV}$ regardless of whether LDM is a Dirac or Majorana fermion. Since the presence of charged particles much lighter than $\sim 100 \mathrm{GeV}$ has been excluded by LEP data, one readily sees that fermionic LDM particles cannot explain the 511 $\mathrm{keV}$ line emission unless one considers couplings at the edge of perturbativity.

Our results also indicate that a $Z^{\prime}$ cross-section cannot explain the observed $511 \mathrm{keV}$ emission on its own. A cross-section strictly proportional to $v^{2}$ can be ruled out by $\Delta_{\text {MLR }} \geqslant 29.9$. The best-fitting fluxes obtained for a linear combination of the models with an $a$ - and a $b$-term are given in Table 4 where two free parameters have been adjusted to match the SPI data. Although the MLR is somewhat improved, a non-physical solution (with one of the factors being negative) is always obtained, suggesting that the bosonexchange channel plays only a minor role within the Milky Way halo.

The existence of scalar DM coupled to heavy (fermionic) particles is thus required for our model in order to explain 


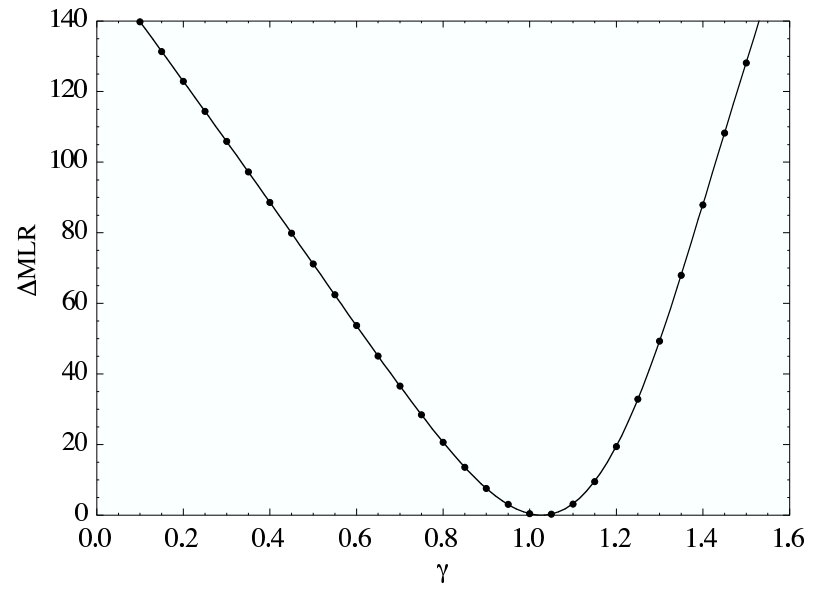

Figure 4. $\Delta_{M L R}=\operatorname{MLR}(\gamma)-\operatorname{MLR}(1)$ for different central asymptotic slopes $\gamma$. Continuous line plots the best-fitting ninthorder polynomial.

the $511 \mathrm{keV}$ line, at variance with the results reported by Boehm et al. (2004) where the $F$ were thought to be facultative, but in agreement with Boehm \& Ascasibar (2004). The reason for the discrepancy resides in the more accurate description of the velocity dispersion profile of the Milky Way.

For the density profiles considered in the present work, decaying dark matter is completely incompatible with the observed morphology of the $511 \mathrm{keV}$ emission. Even for the best-fitting model, M99, decaying dark matter of any sort can be ruled out by $\Delta_{\text {MLR }}=127.9$. It would be possible, though, that a steeper dark matter halo (e.g. as predicted from adiabatic contraction) may provide a better match to the observations (see e.g. Prada et al. 2004).

\subsection{The Milky Way density profile}

All of the configurations in Table 3 display $\Delta_{\text {MLR }}>20$ when compared to our best-fitting model, namely a NFW halo of annihilating dark matter with approximately constant cross-section. We will now assume that this is indeed the nature of dark matter particles. If most galactic positrons did arise from DM annihilations, the spatial distribution of the $511 \mathrm{keV}$ emission line would provide an extremely sensitive probe of the shape of Milky Way dark halo.

We plot in Figure 4 the MLR of a series of models in which the parameters $\alpha$ and $\beta$ in expression (3) have been set to $\alpha=1$ and $\beta=3$, whereas the inner slope $\gamma$ varies from $\gamma=0.1$ to $\gamma=1.6$ in uniform steps $\Delta \gamma=0.05$. We have normalized the MLR to the particular case $\gamma=1$, which corresponds to the NFW profile. Fitting the data points with a ninth-order polynomial, the optimal value of the logarithmic slope is found to be $\gamma=1.03 \pm 0.04$, where the errors have been estimated by equating $\Delta_{\mathrm{MLR}}=1$.

This result is perfectly compatible with $\gamma=1$, but any of the other profiles suggested in the literature would be extremely hard to reconcile with the INTEGRAL/SPI data. This is again at odds with Boehm et al. (2004), where a shallower profile with $\gamma \sim 0.6$ was favoured, based on a coarser comparison between the theoretical predictions and the observed flux and extension of the emission.
Finally, we would like to stress that the constraint we obtain for the inner asymptotic slope of the density profile is so tight that, if the Milky Way dark halo was found to follow a different shape by some independent means, the possibility that dark matter annihilations were the main source of galactic positrons would seem rather unlikely. Systematic effects (see Section 6.1) would in general tend to yield values of $\gamma$ below the real one, so our estimate should be regarded, to a certain extent, as a lower limit. If DM is responsible for the $511 \mathrm{keV}$ emission, $\gamma \gtrsim 1$. If $\gamma<1$, galactic positrons must come from a different physical process.

\section{DISCUSSION}

\subsection{Systematic effects}

The morphology of the galactic $511 \mathrm{keV}$ line emission provides a wealth of information on both the nature of dark matter and the shape of the Milky Way dark halo. We have shown in the previous section that several constraints can be derived for the parameters that characterize dark matter particles (with special emphasis on the LDM scenario), as well as the inner logarithmic slope of the radial density profile.

One should keep in mind that our analysis is based on several simplifying assumptions. Relaxing one (or each) of them would have a different systematic effect on our results:

First, we have neglected any astrophysical contribution to the galactic positron budget. Emission from sources other than dark matter would lower our estimate of $a_{26} m_{\mathrm{MeV}}^{-2}$, equation (17), by a factor proportional to the fraction of DM-related positrons. The effect on the density profile derived for the Milky Way dark halo depends on the spatial distribution of the other sources. Our estimate of the inner slope $\gamma$ would be biased low if the latter was flat, and high if the sources were concentrated near the centre.

Second, the fact that positrons may travel a certain distance before losing their energy and annihilating would flatten the expected emission. Therefore, a steeper density profile would be required in order to fit the observations (i.e. our estimate of $\gamma$ would be biased low). The best-fitting flux would be somewhat lower, so the real value of $a_{26} m_{\mathrm{MeV}}^{-2}$ would again be lower than (17)

Third, our model of the Milky Way is overly simplistic in several respects. On one hand, it is well known that the dark matter haloes found in numerical simulations display a significant degree of triaxiality (Jing \& Suto 2002), although the inclusion of gas cooling tends to yield more spherical haloes (Kazantzidis et al. 2004). In our galaxy, observations of the Sagittarius tidal stream have been interpreted as favouring a spherical halo (Ibata et al. 2001; Majewski et al. 2003), but recent analyses also suggest both oblate (Martínez-Delgado et al. 2004) and prolate (Helmi 2004) shapes. Although the INTEGRAL/SPI data is consistent with spherical symmetry, higher-resolution observations would be needed in order to quantitatively address this issue.

In addition, some substructure is expected to be present in the dark matter halo of our galaxy, both in real and phase space (i.e. the six-dimensional space of positions and velocities). In real space, dark matter clumps would tend 
to boost the expected emission due to the increase in local density (Bergström et al. 1999). It has been argued (e.g. Hooper et al. 2004) that the $511 \mathrm{keV}$ line could actually be detected not only from the galactic centre, but also from the nearest dwarf spheroidals. On the other hand, structures in phase space (such as tidal streams) would not have any effect on the emission through the $a$-channel, but they may have a significant impact both on direct detection experiments (Helmi et al. 2002) and on the emission arising from the $b$-term of the cross-section, mostly because of the increase in dark matter velocity with respect to the local velocity dispersion. Although it does not seem likely, for $b_{26} \sim 10$, that structures in velocity space yield a detectable signature, there might be a mild enhancement in the signal from local dwarfs, particularly near their pericentre.

A more realistic model of the density and velocity distribution of dark matter particles within the Milky Way halo would be given by the results of N-body simulations. Although extremely promising, this approach (see e.g. Stoehr et al. 2003) must face the problem of numerical convergence (i.e. lack of resolution) in order to provide conclusive results.

\subsection{Compatibiliy with low-energy gamma rays}

DM annihilations produce charged particles which, in turn, produce low-energy gammas. But low-energy photons are also expected through the final state radiation (FSR) mechanism or DM annihilations into two monoenergetic photons (with an energy $E=m_{\mathrm{dm}} c^{2}$ ). In principle, the observation of such line would be an unambiguous signature of the LDM scenario. However, the corresponding cross-section relies on a box diagram that involves four powers of the electric charge $e$, and therefore it is expected to be suppressed compared to the FSR mechanism in which either the electron, the positron or the charged particle that is exchanged during the annihilation emits a photon.

A rough estimate of the gamma ray flux generated by FSR was given in Boehm et al. (2004). In an attempt to build a model that would surely satisfy astrophysical constraints, the authors assumed that the FSR cross-section was as large as the annihilation cross-section. The electric charge (which is expected to reduce the FSR cross-section by a factor $e^{2}$ ) was therefore deliberately omitted in order to obtain the maximal flux of low-energy gamma rays that LDM could produce. Comparing this estimate with observations, Boehm et al. (2004) deduced that particles lighter than 100 $\mathrm{MeV}$ were excluded unless their annihilation cross-section was suppressed in our galaxy with respect to its value in the primordial universe, which led the authors to postulate the existence of a velocity-dependent annihilation cross-section.

The required suppression factor turns out to be consistent with that obtained from the analysis of the $511 \mathrm{keV}$ line. More precisely, a cross-section as large as equation (17) does not overproduce gamma rays if the DM particles are lighter than $30 \mathrm{MeV}$. It does, in principle, if DM is heavier, but given the drastic assumption on the FSR cross-section, it was considered that even $100 \mathrm{MeV}$ particles would both explain the $511 \mathrm{keV}$ line and be compatible with gamma-ray observations.

In a recent paper, Beacom et al. (2005) claimed that particles heavier than $20 \mathrm{MeV}$ were actually not consistent with the gamma-ray data. To make this point, they wrote the FSR cross-section as

$\frac{\mathrm{d} \sigma_{\mathrm{b}}}{\mathrm{d} E_{\gamma}}=\sigma_{\mathrm{ann}} \times R_{\text {corr }}(E)$

and used $R_{\text {corr }}(E)=\frac{\alpha}{\pi} \frac{1+\left(s^{\prime} / s\right)^{2}}{E_{\gamma}}\left[\ln \left(\frac{s^{\prime}}{m_{e}^{2}}\right)-1\right]$, with $s \equiv 4 m_{\mathrm{dm}}$ and $s^{\prime} \equiv 4 m_{\mathrm{dm}}\left(m_{\mathrm{dm}}-E_{\gamma}\right)$, as computed by Berends \& Bohm (1987) for the radiative correction to $e^{+} e^{-} \rightarrow \mu^{+} \mu^{-}$.

To our knowledge, it has never been proven that any radiative cross-section can be written in the same way as $e^{+} e^{-} \rightarrow \mu^{+} \mu^{-} \gamma$ in the hard photon limit, so it might be possible that equation (25) does not provide a completely accurate description of the radiative corrections to the annihilation process. Nonetheless, a detailed computation would be quite delicate, and it certainly lies well beyond the scope of the present work. We will therefore estimate the gamma ray flux according to the procedure followed by Beacom et al. (2005). Assuming the same $R_{\text {corr }}(E)$, the intensity of internal bremsstrahlung gamma rays at the earth is related to the $511 \mathrm{keV}$ skymaps by

$\frac{\mathrm{d} I_{\mathrm{b}}}{\mathrm{d} E_{\gamma}}=\frac{I_{511}}{0.605} \frac{\alpha}{\pi} \frac{1+\left(s^{\prime} / s\right)^{2}}{E_{\gamma}}\left[\ln \left(\frac{s^{\prime}}{m_{e}^{2}}\right)-1\right]$.

Comparison with COMPTEL and EGRET data only requires the computation of the average intensity over the appropriate region of the sky. Using the model that best fits the $511 \mathrm{keV}$ line, i.e. a NFW profile with constant crosssection given by equation (17), we obtain

$\left\langle I_{511}\right\rangle_{|l|<30^{\circ},|b|<5^{\circ}}=\frac{\Phi_{511}}{\Delta \Omega} \approx 0.005 \mathrm{~cm}^{-2} \mathrm{~s}^{-1} \mathrm{sr}^{-1}$.

Even if we restrict to the innermost $5^{\circ}$ in both longitude and latitude, our expectation for the average intensity

$\left\langle I_{511}\right\rangle_{|l|<5^{\circ},|b|<5^{\circ}} \approx 0.018 \mathrm{~cm}^{-2} \mathrm{~s}^{-1} \mathrm{sr}^{-1}$

is somewhat lower than the estimate used by Beacom et al. (2005).

The solid angle considered and the precise shape of the energy dependence of the observational data have important consequences on the inferred constraints. Figure 5 shows the limits we obtain for the mass of the dark matter candidate. The factor of $\sim 4$ difference between equations (27) and (28) translates into a factor of 3 for the maximum dark matter mass, i.e. $m_{\mathrm{dm}} \lesssim[30-100] \mathrm{MeV}$. This constraint is similar (though less severe) to that obtained by Beacom et al. (2005), and it also agrees roughly with the results reported by Boehm et al. (2004) despite the fact that much less than 2 photons are predicted by integrating equation (26) over the photon energy (e.g. between 1 and $100 \mathrm{MeV}$ ). However, the constrain provided by the 'spectrum' of the observed gamma-ray radiation is much more stringent than its total flux (or, more precisely, the flux above $E_{\gamma}^{\min } \leqslant m_{\mathrm{dm}} c^{2}$, see Boehm et al. 2004).

In any case, we would also like to emphasize that a rigorous comparison with COMPTEL and EGRET data, analogous to the analysis of the $511 \mathrm{keV}$ line presented here, would be necessary in order to provide accurate constrains on the mass range allowed for the dark matter particles. 


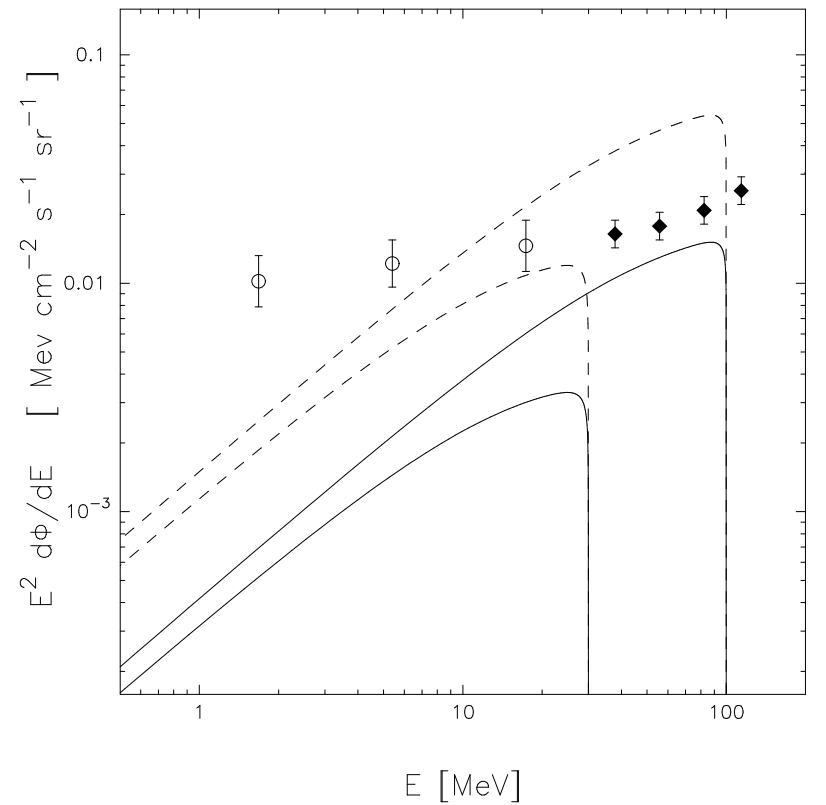

Figure 5. Comparison with COMPTEL (circles) and EGRET (diamonds) data (Strong et al. 2000). Lines plot the gamma ray flux expected for $m_{\mathrm{dm}}=30$ and $100 \mathrm{MeV}$, according to expression 26] Solid and dashed lines correspond to the regions $|b|<5^{\circ}$, $|l|<30^{\circ}$ 27] and $|b|<5^{\circ},|l|<5^{\circ}$ [28.

\subsection{Tests of the LDM model in particle physics experiments}

We mentioned in a previous section that the $F$ particles could be detectable in the next colliders. The $F$ particles could be detected through their two-body decay $F_{p}{ }^{-} \rightarrow p^{-}+$ $\mathbb{E}$, where $\mathbb{E}$ denotes the missing energy associated with Dark Matter and $p^{-}$the Standard Model particle associated with $F_{p}$ (for example $F_{e}{ }^{-} \rightarrow e^{-}+\not \mathbb{E}$ ). The couplings are large enough to allow for the decay within the detector, although the production cross-section of $F$ particles $\left(p^{+} p^{-} \rightarrow F_{p}^{-} F_{p}^{+}\right)$ may be too small to yield a visible signature. This crosssection can reach, however, a few pb for $m_{F p} \sim 100 \mathrm{GeV}$, $m_{\mathrm{dm}} \sim 100 \mathrm{MeV}$ and couplings $F_{p}-p-d m$ of the order of (20). The presence of $F_{q}$ particles could then be detected at LHC through the modification of the total hadronic crosssection (if either of the couplings $c_{l}$ or $c_{r}$ remains relatively large).

The $F_{e}$ decay should be quite similar to the (supersymmetric) decay of a chargino into an electron and a sneutrino. However, in our case the mass of the DM particle is much smaller than the sneutrino mass. Hence, we expect a fair repartition of energy between the electron and the missing energy in the LDM scenario. This should be quite different in a supersymmetric framework, albeit more precise estimates would depend on the difference of mass between the sneutrino and the chargino (and therefore on the model considered). In any case, a possible way to discriminate between our scenario and supersymmetric particles could be the absence of other signatures. For example, unlike the chargino which is expected to also decay into a selectron and a neutrino, the $F_{e}$ particles are assumed to leave only one main signature (i.e. the decay into an electron and missing energy). The precise relationship between the $F_{e}$ mass and the couplings (20) should also help in discriminating between the LDM and supersymmetric scenarios.

Dark matter production through $p^{+} p^{-} \rightarrow \mathrm{dm} d m$ could in principle be detectable in the initial state radiation process (where a photon is emitted by the particles of the initial state). However, the associated cross-section is expected to be smaller than a few fb even for $m_{\mathrm{dm}} \sim 100 \mathrm{MeV}$, and is therefore less interesting.

There are two other tests that could be really crucial for our purpose. One is based on the so-called $\mathrm{NuTeV}$ anomaly and the other one on the value of the fine structure constant $\alpha$.

$\mathrm{NuTeV}$ is an experiment which measured the ratio

$$
R=\frac{\text { neutral currents }}{\text { charged currents }}=\left(g_{l}^{2}-g_{r}^{2}\right),
$$

with $g_{l, r}^{2}=\left[\left(g_{l, r}^{u}\right)^{2}+\left(g_{l, r}^{d}\right)^{2}\right] / 4$ and $g_{l, r}^{f}=2\left(T_{3}\left(f_{l, r}\right)-\right.$ $\left.Q(f) \sin \theta_{W}^{2}\right)$ the left and right couplings of the $Z$ boson to fermions. Through the measurement of this ratio, one can infer the value of the mixing angle $\sin \theta_{W}^{2}$ and compare it to Standard Model expectations (obtained notably from LEP electroweak precision observables). Surprisingly, NuTeV result for $\sin \theta_{W}^{2}$ turns out to be slightly discrepant with the Standard Model value (McFarland et al. 2003; Naples et al. 2003; McFarland \& Moch 2003).

There are several possible explanations to the $\mathrm{NuTeV}$ anomaly. In particular, isospin violation and/or strange sea asymmetry as well as other effects such as electroweak corrections may reduce significantly the discrepancy. However, at present, the situation is still uncertain and there is an open window for new physics. The best explanation to that respect turns out to be a light gauge boson (Davidson et al. 2002; Boehm 2004), similar to the one we introduce in the LDM scenario. If the $\mathrm{NuTeV}$ anomaly disappears or is significantly reduced, then one could set a limit on the $Z^{\prime}$ couplings to ordinary matter. On the other hand, more evidence in favour of a light $Z^{\prime}$ would certainly give more credit to the LDM scenario.

The other test concerns the existence of heavy particles (Boehm \& Ascasibar 2004). We expect them to contribute to the electron anomalous magnetic moment as: $\delta a_{\mu, e} \sim$ $\frac{c_{l} c_{r}}{16 \pi^{2}} \frac{m_{\mu, e}}{m_{F_{\mu, e}}}>0$. Using expression (20), we obtain

$\delta a_{e}^{F} \sim 5.41 \times 10^{-12} \frac{m_{\mathrm{dm}}}{\mathrm{MeV}}$.

It turns out that there is a small discrepancy between the theoretical value of $a_{e}$ (hereafter denotes $a_{t h}$ ) and its measurement $\left(a_{\text {exp }}\right)$ :

$\Delta a_{e}=\left(a_{\text {exp }}-a_{t h}\right) \sim(3.44-3.49) 10^{-11}$

where the first number is obtained from the positron g2 , while the second one is from the electron. We estimate (30) by using $a_{t h}=f(\alpha)$ with $\alpha=\alpha_{Q H}$, the fine structure constant as measured by Quantum Hall effect $(\mathrm{QH})$ experiments. There are other experiments aiming at measuring $\alpha$, but QH experiments seem the most precise at present (see e.g. Kinoshita 1996).

Usually, one assumes instead the validity of QED and imposes that $a_{t h} \equiv a_{Q E D}$ matches $a_{\exp }$. One then gets a 'theoretical' estimate of $\alpha$ that is extremely precise and in fact the most significant input in the $\alpha$ value given in the CODATA. The latter differs too much from $\alpha_{Q H}$ for the dif- 
ference to be explained by common extensions of the Standard Model such as supersymmetry, but experiments measuring $\alpha$ did not reach the sensitivity of $g-2$ experiments as yet. Therefore, there is the hope that the difference may eventually go away and that there is no new contribution other than QED.

However, the discrepancy between $\alpha_{Q H}$ and $\alpha$ could originate from new physics. In particular, the introduction of heavy particles coupled to light scalars adds a new contribution in $a_{t h}$ which is greater than expected in e.g. supersymmetry, due to the DM mass scale.

Including this new contribution $\left(\delta a_{e}^{F}\right)$, one obtains $\alpha \simeq$ $\alpha_{Q H}\left(\right.$ or $\left.a_{t h} \simeq a_{\text {exp }}\right)$ if $m_{\mathrm{dm}} \sim 6.4 \mathrm{MeV}$, using the average value of $a_{t h}$ computed with $\alpha_{Q H}$ (see Kinoshita 1996). Taking into account theoretical and experimental uncertainties, $m_{\mathrm{dm}} \sim 3-9 \mathrm{MeV}$. For smaller DM masses, we obtain $\delta a_{e}^{F} \leqslant \Delta a_{e}$, while for larger DM masses $\delta a_{e}^{F} \geqslant \Delta a_{e}$. As explained above, there is no direct measurement of $\alpha$ that is as precise as the $g-2$ as yet. Therefore, it is hard to exclude values above $7 \mathrm{MeV}$. However, this certainly places a very strong contraint and motivates further experiments measuring the value of the fine structure constant directly (and independently of QED). If these experiments find a perfect agreement with the value recommended in the CODATA, then the LDM scenario will have difficulties in explaining the $511 \mathrm{keV}$ line emission. If they found a discrepancy (whether it is positive or negative) then LDM will remain a serious candidate because it would be the sign of new physics. In particular, if the value $\alpha_{Q H}$ is confirmed, then the LDM scenario may reconcile the results from both $g-2$ and $\alpha$ experiments, despite the difference of sensitivity.

Measurements of the fine structure interval of $H_{e}$-like ions based on laser spectroscopy might also provide a very precise determination of $\alpha$ if theory gets as accurate as the experimental determinations (see e.g. Pachucki \& Sapirstein 2003, and references therein). However, this method assumes that there is no additional contribution from new physics, so it may not be suitable for answering whether the $F$ particles exist or not.

Taking the same couplings and the same mass $m_{F}$ for $F_{e}$ as for $F_{\mu}$, we obtain a very large contribution to the muon $g-2$. Our prediction, in fact, exceeds the experimental value by a factor $2-3$, which is itself larger than the Standard Model prediction (Bennett et al. 2004). It was found $\Delta a_{\mu}=\left(a_{\exp }-a_{t h}\right) \in[1.6,2.7] 10^{-9}$. So, by using $m_{\mathrm{MeV}} \sim 6-7$ and $m_{F \mu}=3 m_{F e}$ (or e.g. $m_{F \mu}=2 m_{F e}$ and smaller couplings to the muons), our prediction for the muon $g-2$ becomes compatible with the experimental value. In fact, the LDM scenario would even explain the well-known discrepancy. Note that such a hierarchy exists in the Standard Model and it is very realistic to assume that it exists also in any other extensions.

Hence, the LDM scenario could in fact explain both the experimental values of the fine structure constant and the muon $g-2$ for $m_{\mathrm{dm}} \approx 6-7 \mathrm{MeV}$.

\section{CONCLUSIONS}

In this paper, we use the intensity and morphology of the observed $511 \mathrm{keV}$ line to put independent constraints on the nature of LDM and the shape of the Milky Way dark halo. Our main assumptions are that DM decays or annihilations are the main source of galactic positrons and that these positrons do not travel long distances before annihilating. Theoretical expectations for the flux distribution are computed for different DM models and galactic density profiles. The sky maps thus obtained are then convolved with the SPI response function and used as a source for the INTEGRAL model-fitting analysis.

We can rule out from a likelihood analysis the possibility that decaying dark matter is responsible for the observed emission, unless the density profile of the Milky Way dark halo turns out to be extremely cuspy (with inner asymptotic slope $\gamma>1.5)$.

We can exclude fermionic LDM particles, because it would require the introduction of charged scalars lighter than $100 \mathrm{GeV}$, which should have already been detected in past colliders. As a result, LDM is likely to be a scalar.

For annihilating scalar LDM, it is shown that the exchange of a heavy fermion $\left(F_{e}\right)$ is required in order to fit the morphology of the $511 \mathrm{keV}$ line, while the existence of a $Z^{\prime}$ boson would be necessary to satisfy the relic density criterion. Assuming a full spectrum and, most precisely, the existence of $F$ particles associated with quarks $\left(F_{q}\right)$, we notice that there might be a signature at the Large Hadron Collider, notably through the measure of the total hadronic cross-section and the two body decay of these $F_{q}$ particles.

However, the most promising signature of $F$ particles turns out to be their contribution to the electron $g-2$. The new contribution would make the measurements of the fine structure by the Quantum Hall experiment and the electron anomalous magnetic moment compatible for $m_{\mathrm{dm}} \sim 6-$ $7 \mathrm{MeV}$, meaning that the value of $\alpha$ quoted in the CODATA (and used for many estimates) may not be the correct one. The dark matter mass could however be larger than $\sim 6$ $\mathrm{MeV}$. The existence of clumps, the fact that the dark halo is probably not perfectly spherical and the contribution to the positron population from astrophysical sources are all expected to decrease our estimate in equation [17 therefore allowing for larger dark matter masses.

Assuming the existence of this spectrum (and $F_{\mu}$ particles), we also find a non-negligible contribution to the muon $g-2$. Both $F_{e}$ and $F_{\mu}$ could then explain the discrepancy between the Standard Model predictions and the experimental values of the muon $g-2$ and the fine structure constant. Alternatively those could provide a way to constrain the LDM scenario.

Concerning the shape of the Milky Way dark halo, our results clearly indicate that dark matter particles can only explain the observed $511 \mathrm{keV}$ emission if our galaxy features a cuspy density profile. For any annihilating DM candidate with constant cross-section, the best-fitting inner asymptotic slope is found to be $\gamma=1.03 \pm 0.04$.

To sum up, we would like to stress the fact that the 511 $\mathrm{keV}$ emission line provides extremely stringent constraints on the light dark matter parameters. Independent confirmations are needed to prove that dark matter contributes to most of the galactic positrons. Such confirmation might come either from the lack of astrophysical sources and/or from detection in particle physics experiments. Observations of the density profile of the Milky Way have the possibility to rule out a dark-matter related origin of galactic positrons if the density profile of our galaxy is found to be shallow 
at the centre. Alternatively, the discovery of LDM particles would have a tremendous impact on the determination of the dark halo profile of the Milky Way.

\section{ACKNOWLEDGMENTS}

The authors would like to thank J. Devriendt, F. Ferrer, P. Janot and P. Salati for useful discussions. Y. Ascasibar acknowledges support from NASA grants G02-3164X and G04-5152X.

\section{REFERENCES}

Ahn K., Komatsu E., 2005, Phys. Rev., D71, 021303

Alcock C., et al., 2000, ApJ, 541, 734

Ascasibar Y., Yepes G., Gottlöber S., Müller V., 2004, MNRAS, 352, 1109

Beacom J. F., Bell N. F., Bertone G., 2005, Physical Review Letters, 94, 171301

Bennett G. W., et al., 2004, Phys. Rev. Lett., 92, 161802

Berends F. A., Bohm A., 1987

Bergström L., Edsjö J., Gondolo P., Ullio P., 1999, Phys. Rev. D, 59, 043506

Bertschinger E., 1985, ApJS, 58, 39

Binney J., 2004a, in IAU Symposium 220 Conference Summary (astro-ph/0310219)

Binney J., 2004b, MNRAS, 350, 939

Binney J. J., Evans N. W., 2001, MNRAS, 327, L27

Blumenthal G. R., Faber S. M., Flores R., Primack J. R., 1986, ApJ, 301, 27

Boehm C., 2004, Phys. Rev., D70, 055007

Boehm C., Ascasibar Y., 2004, Phys. Rev., D70, 115013

Boehm C., Ensslin T. A., Silk J., 2004, J. Phys., G30, 279

Boehm C., Fayet P., 2004, Nucl. Phys., B683, 219

Boehm C., Hooper D., Silk J., Casse M., Paul J., 2004, Physical Review Letters, 92, 101301

Churazov E., Sunyaev R., Sazonov S., Revnivtsev M., Varshalovich D., 2005, MNRAS, 357, 1377

Davidson S., Forte S., Gambino P., Rius N., Strumia A., 2002, JHEP, 02, 037

de Blok W. J. G., 2005, (astro-ph/0506753)

Diemand J., Moore B., Stadel J., Kazantzidis S., 2004, MNRAS, 348, 977

Fillmore J. A., Goldreich P., 1984, ApJ, 281, 1

Flores R. A., Primack J. R., 1994, ApJ, 427, L1

Gentile G., Salucci P., Klein U., Vergani D., Kalberla P., 2004, MNRAS, 351, 903

Gondolo P., Silk J., 1999, Phys. Rev. Lett., 83, 1719

Gunn J. E., 1977, ApJ, 218, 592

Gunn J. E., Gott J. R. I., 1972, ApJ, 176, 1

Harris M. J., Teegarden B. J., Cline T. L., Gehrels N., Palmer D. M., Ramaty R., Seifert H., 1998, ApJ, 501, L55+

Hayashi E., Navarro J. F., Jenkins A., Frenk C. S., Power C., White S. D. M., Springel V., Stadel J., Quinn T., Wadsley J., 2005, ApJ, submitted (astro-ph/0408132)

Hayashi E., Navarro J. F., Power C., Jenkins A., Frenk C. S., White S. D. M., Springel V., Stadel J., Quinn T. R., 2004, MNRAS, 355, 794

Helmi A., 2004, ApJ, 610, L97
Helmi A., White S. D., Springel V., 2002, Phys. Rev. D, 66, 063502

Hooper D., Ferrer F., Boehm C., Silk J., Paul J., Evans N. W., Casse M., 2004, Physical Review Letters, 93, 161302

Hooper D., Wang L.-T., 2004, Phys. Rev., D70, 063506

Ibata R., Lewis G. F., Irwin M., Totten E., Quinn T., 2001, ApJ, 551, 294

Jean P., et al., 2003, A\&A, 407, L55

Jing Y. P., Suto Y., 2002, ApJ, 574, 538

Johnson W. N., Harnden F. R., Haymes R. C., 1972, ApJ, $172, \mathrm{~L} 1+$

Kazantzidis S., Kravtsov A. V., Zentner A. R., Allgood B., Nagai D., Moore B., 2004, ApJ, 611, L73

Kinoshita T., 1996, Rept. Prog. Phys., 59, 1459

Kinzer R. L., Milne P. A., Kurfess J. D., Strickman M. S., Johnson W. N., Purcell W. R., 2001, ApJ, 559, 282

Knödlseder J., Jean P., Lonjou V., Weidenspointner G., Guessoum N., Gillard W., Skinner G., von Ballmoos P., Vedrenne G., Roques J.-P., Schanne S., Teegarden B., Schönfelder V., Winkler C., 2005, A\&A, 441, 513

Lee B. W., Weinberg S., 1977, Phys. Rev. Lett., 39, 165

Leventhal M., MacCallum C. J., Stang P. D., 1978, ApJ, 225, L11

McFarland K. S., et al., 2003, Int. J. Mod. Phys., A18, 3841 McFarland K. S., Moch S.-O., 2003, (hep-ph/0306052)

Majewski S. R., Skrutskie M. F., Weinberg M. D., Ostheimer J. C., 2003, ApJ, 599, 1082

Martínez-Delgado D., Gómez-Flechoso M. Á., Aparicio A., Carrera R., 2004, ApJ, 601, 242

Moore B., 1994, Nature, 370, 629

Moore B., et al., 1999, ApJ, 524, L19

Naples D., et al., 2003, Nucl. Phys. Proc. Suppl., 118, 164

Navarro J. F., Frenk C. S., White S. D. M., 1997, ApJ, 490, 493

Navarro J. F., Hayashi E., Power C., Jenkins A. R., Frenk C. S., White S. D. M., Springel V., Stadel J., Quinn T. R., 2004, MNRAS, 349, 1039

Pachucki K., Sapirstein J., 2003, J. Phys. B, 36, 803

Picciotto C., Pospelov M., 2005, Phys. Lett., B605, 15

Power C., Navarro J. F., Jenkins A., Frenk C. S., White S. D. M., Springel V., Stadel J., Quinn T., 2003, MNRAS, 338, 14

Prada F., Klypin A., Flix J., Martínez M., Simonneau E., 2004, Physical Review Letters, 93, 241301

Rasia E., Tormen G., Moscardini L., 2004, MNRAS, 351, 237

Stoehr F., White S. D. M., Springel V., Tormen G., Yoshida N., 2003, MNRAS, 345, 1313

Strong A. W., Moskalenko I. V., Reimer O., 2000, ApJ, 537,763

Sumi T., Wozniak P. R., Udalski A., Szymanski M., Kubiak M., Pietrzynski G., Soszynski I., Zebrun K., Szewczyk O., Wyrzykowski L., Paczynski B., 2005, (astro-ph/0502363)

Taylor J. E., Navarro J. F., 2001, ApJ, 563, 483 